\title{
O impacto da pandemia do Covid-19 na saúde mental dos profissionais médicos de um hospital referência para tratamento do Covid-19 em uma capital do nordeste
}

\author{
The impact of the Covid-19 pandemic on the mental health of medical professionals at a referral \\ hospital for Covid-19 treatment in a northeastern capital \\ El impacto de la pandemia de Covid-19 en la salud mental de los profesionales médicos en un \\ hospital de referencia para el tratamiento de Covid-19 en una capital del noreste
}

Recebido: 09/12/2021 | Revisado: 14/12/2021 | Aceito: 16/12/2021 | Publicado: 01/01/2022

\author{
Caroline Andrade Sousa \\ ORCID: https://orcid.org/0000-0002-2232-5784 \\ Centro Universitário UNINOVAFAPI, Brasil \\ E-mail: andradesousacaroline@gmail.com \\ Ravana Correia Araújo \\ ORCID: https://orcid.org/0000-0002-3732-7010 \\ Centro Universitário UNINOVAFAPI, Brasil \\ E-mail: ravanelck@gmail.com \\ Juliana Bandeira da Rocha Lima \\ ORCID: https://orcid.org/0000-0002-3822-2181 \\ Universidade Federal do Piauí, Brasil \\ E-mail: julianabrlima@gmail.com \\ Jyselda de Jesus Lemos Duarte \\ ORCID: https://orcid.org/0000-0003-4835-9816 \\ Centro Universitário UNINOVAFAPI, Brasil \\ E-mail: jys.1.duarte@hotmail.com
}

\begin{abstract}
Resumo
Objetivo: analisar o impacto da pandemia do Covid-19 na saúde mental de profissionais médicos em um hospital público em Teresina-PI. Metodologia: estudo transversal, descritivo e quantitativo com finalidade básica estratégica. A população objeto da pesquisa foi constituída por 29 médicos e utilizou-se para coleta de dados um questionário com 10 perguntas com as variáveis: gênero, idade, raça, área de atuação na medicina e tempo, treinamento em paramentação e desparamentação, diagnóstico prévio de insônia, ansiedade ou depressão e se faz tratamento e uma escala likert de 5 pontos para avaliação dos fatores estressores, baseada naqueles mais relatados na literatura. Ademais, foram aplicadas escalas para triagem de transtorno de estresse pós- traumático (PC-PTSD), transtorno de ansiedade generalizada (GAD2) e depressão (PHO-2), validadas na literatura. Resultados: foram colhidas 29 respostas, sendo 10 do sexo feminino e 19 do sexo masculino. A maioria tinha entre 20 e 30 anos (41,4\%), com atuação como clínico (51,7\%). 55\% tiveram triagem positiva para ansiedade, $45 \%$ para transtorno pós traumático e $45 \%$ para depressão. Para controle do estresse, atividade física regular e religião e/ou espiritualidade (34,5\%) foram as mais descritas. Os fatores estressores mais prevalentes foram ter familiares no grupo de risco e a escassez de insumos e leitos (75\%). Conclusão: Esses resultados apontam para a necessidade de implementação de políticas efetivas de saúde mental, de modo a proteger o bem estar mental e físico desses profissionais visando, também, a permanente assistência de qualidade à população.
\end{abstract}

Palavras-chave: Saúde mental; COVID-19; TEP; Ansiedade; Depressão.

\begin{abstract}
Objective: to analyze the impact of the Covid-19 pandemic on the mental health of medical professionals in a public hospital in Teresina-PI. Methodology: cross-sectional, descriptive and quantitative study with strategic basics. The population object of the research was found by 29 physicians and a questionnaire with 10 questions was used for data collection, with the variables: gender, age, race, area of expertise in medicine and time, training in garments and undressing, previous diagnosis of insomnia, anxiety or depression, and treatment is performed using a 5-point scale to assess stressors, based on those most reported in the literature. Furthermore, scales were applied to screen for posttraumatic stress disorder (PC-PTSD), generalized anxiety disorder (GAD-2) and depression (PHO-2), validated in the literature. Results: 29 responses were collected, 10 female and 19 male. Most were between 20 and 30 years old $(41.4 \%)$, working as a clinician $(51.7 \%) .55 \%$ were screened positive for anxiety, $45 \%$ for post-traumatic disorder and $45 \%$ for depression. For stress control, regular physical activity and religion and/or spirituality (34.5\%) were the oldest. The most prevalent stressors were having family members in the risk group and the lack of supplies and beds (75\%).
\end{abstract}


Conclusion: These results point to the need to implement effective mental health policies, in order to protect the old mental and physical well-being, as well as the permanence of quality to the population.

Keywords: Mental health; COVID-19; TEP; Anxiety; Depression.

\section{Resumen}

Objetivo: analizar el impacto de la pandemia Covid-19 en la salud mental de los profesionales médicos de un hospital público de Teresina-PI. Metodología: estudio transversal, descriptivo y cuantitativo con fundamentos estratégicos. La población objeto de la investigación fue encontrada por 29 médicos y se utilizó un cuestionario con 10 preguntas para la recolección de datos, con las variables: sexo, edad, raza, área de especialización en medicina y tiempo, entrenamiento en vestuario y desvestimiento, previo El diagnóstico de insomnio, ansiedad o depresión y el tratamiento se realiza mediante una escala de 5 puntos para evaluar los factores estresantes, en base a los más reportados en la literatura. Además, se aplicaron escalas para el cribado de trastorno de estrés postraumático (PC-PTSD), trastorno de ansiedad generalizada (GAD-2) y depresión (PHO-2), validadas en la literatura. Resultados: Se recolectaron 29 respuestas, 10 femeninas y 19 masculinas. La mayoría tenía entre 20 y 30 años $(41,4 \%)$ y trabajaba como clínico $(51,7 \%)$. El 55\% resultó positivo para ansiedad, el $45 \%$ para trastorno postraumático y el $45 \%$ para depresión. Para el control del estrés, la actividad física habitual y la religión y / o espiritualidad (34,5\%) fueron las más antiguas. Los estresores más prevalentes fueron tener familiares en el grupo de riesgo y la falta de suministros y camas (75\%). Conclusión: Estos resultados apuntan a la necesidad de implementar políticas de salud mental efectivas, a fin de proteger el antiguo bienestar mental y físico, así como la permanencia de la calidad para la población.

Palabras clave: Salud mental; COVID-19; TEP; Ansiedad; Depresión.

\section{Introdução}

Ao final do ano de 2019, a cidade de Wuhan, localizada na China, presenciou o surgimento de uma nova pneumonia causada pela doença do coronavírus de 2019 (COVID-19), que se espalhou no país e em outras nações. A principal forma de transmissão é por meio de gotículas respiratórias de pessoa a pessoa, por pacientes sintomáticos ou assintomáticos, além da propagação através de fômites. (Lai, et al., 2020; Meyerowitz, 2020).

Segundo relatório da Organização Mundial de Saúde (OMS) (2021), até 08 de novembro de 2021 foram notificados 249.743.428 casos confirmados e 5.047.652 óbitos com casos espalhados por todas as regiões do mundo. O Brasil até dia 08 de novembro de 2021, confirmou 21.886.077 casos e 609.573 mortes. Já no Piauí, foram confirmados até o dia 08 de novembro de 327.325 casos e 7.118 óbitos, sendo 2579 destes na capital Teresina (Ministério da Saúde, 2021; Secretaria de Estado da Saúde, 2021).

Nesse contexto de pandemia, os gestores, profissionais de saúde e cientistas concentraram grandes esforços para compreender esse novo patógeno e os mecanismos fisiopatológicos envolvidos, com o fim de propor medidas de prevenção, contenção e tratamento da doença. Todavia, segundo Orneel (2020), em situações como essa, há uma tendência à negligenciar ou subestimar as implicações psicológicas e psiquiátricas secundárias ao evento, sendo o número de pessoas afetadas por estas, muitas vezes, maior do que a quantidade de pessoas afetadas pela própria infecção. E, embora toda a população esteja suscetível a isso, alguns grupos específicos são especialmente vulneráveis.

Devido à alta infectividade do SARS-COV-2 e, consequentemente, o ascendente número de casos confirmados e suspeitos, uma grande parcela dos profissionais médicos foram realocados para áreas diferentes de sua especialização clínica e tiveram que trabalhar turnos extras para atender a alta demanda de pacientes. Além disso, as altas taxas de mortalidade, a falta de diretrizes seguras sobre tratamentos e vacinas eficazes, as medidas de distanciamento social, a escassez de equipamentos de proteção individual (EPI), a possibilidade de adquirir e transmitir a patologia para familiares e amigos, a exposição diária ao patógeno, entre outros fatores, tornaram esses profissionais que estão na "linha de frente" mais vulneráveis a desenvolver sofrimento psicológico e transtornos psiquiátricos como medo, estresse agudo, ansiedade, depressão e distúrbios do sono (Lai, et al., 2020; Xiang, et al., 2020; Shechter, et al., 2020).

Nesse sentido, analisar a saúde mental de profissionais médicos é relevante não somente no contexto do enfrentamento do COVID-19, mas na promoção de saúde continua a esse grupo, especificamente. Problemas como estresse, ansiedade, sintomas 
depressivos, insônia, medo, raiva e negação não afetam apenas a atenção desses profissionais, a compreensão e a tomada de decisão, mas também podem ter efeitos duradouros em sua própria saúde a longo prazo (Kang, et al., 2020).

Desse modo, esse trabalho tem como objetivo analisar o impacto da pandemia do Covid-19 na saúde mental de profissionais médicos em um hospital público em Teresina-PI, sob a ótica de três principais transtornos: depressão, ansiedade e estresse agudo. Além disso, analisar se houve diferença no nível do impacto entre profissionais médicos do sexo feminino e do sexo masculino, identificar quais foram os possíveis aspectos decorrentes do contexto da pandemia ou de histórico pessoal que desencadearam ou influenciaram o desenvolvimento de alterações mentais e observar se práticas para controle de estresse foram fatores de proteção para o surgimento de alterações mentais.

\section{Metodologia}

Segundo Pereira, et al. (2018), trata-se de um estudo transversal, descritivo e quantitativo com finalidade básica estratégica. A população objeto da pesquisa foi constituída por profissionais médicos do hospital público Dr. Miguel Couto localizado no bairro Monte Castelo no município de Teresina-PI, Brasil. Tendo em vista tratar-se de uma população finita composta por 29 indivíduos, todos esses profissionais foram incluídos na pesquisa, sendo informados sobre o procedimento e os objetivos.

Como instrumento para a coleta de dados, realizada em julho de 2021, foi utilizado um questionário estruturado com 10 perguntas objetivas, nas quais as variáveis foram gênero, idade, raça, área de atuação na medicina e tempo, treinamento em paramentação e desparamentação, diagnóstico prévio de estresse agudo, ansiedade ou depressão e se faz tratamento. Ademais, com o fim de observar se há uma relação entre práticas para controle de estresse (atividade física, espiritualidade, entre outros) e menos alterações mentais, foi adicionada ao questionário uma pergunta sobre esse tema.

Como não há questionários validados para avaliação dos estressores em relação ao Covid-19 em profissionais de saúde, uma escala likert de 4 pontos (1 - Não; 2 - Leve; 3 - Moderado; 4 - Severo) foi desenvolvida pelas pesquisadoras. Teve como base os estressores mais comumente relatados na literatura consultada, principalmente, na pesquisa transversal realizada por Shechter, et al (2020) em Nova York com 657 profissionais de saúde em um grande centro médico.

Para avaliar de forma ampla esses estressores, visando abranger situações decorrentes do contexto da pandemia e de histórico pessoal, a escala likert avaliou fatores relacionados à família e preocupações fora do ambiente clínico (transmitir Covid19 para familiares, ter familiares do grupo de risco, distanciamento social da família e renda familiar), ao ambiente de trabalho (contato com paciente com Covid-19, morte de pacientes e/ou colegas de trabalho, carga horária de trabalho, contrair Covid-19 e realocação para áreas diferentes de sua especialidade clínica) e às diretrizes e políticas nacionais (escassez de insumos e leitos, falta de EPIs e falta de diretrizes de tratamento seguras).

Além disso, as escalas de Triagem de Transtorno de Estresse Pós Traumático (TEPT) para Atenção Primária (PCPTSD), de Transtorno de Ansiedade Generalizada (GAD-2) e Questionário sobre a saúde do paciente (PHO-2) foram aplicadas para triagem de TEPT, ansiedade e depressão, respectivamente. Foram considerados positivos para TEPT aqueles que tiveram pontuação igual ou maior que 3, e aqueles que tiveram pontuação igual ou maior que 2 para a escala GAD-2 e PHO-2 entraram na triagem positiva para ansiedade e depressão, respectivamente. Essas escalas foram escolhidas por serem amplamente utilizadas e por terem sua eficácia e confiabilidade validadas em diversos estudos como Gaya (2011), Castro (2011), Kroenke (2007) e Prins, et al. (2004).

A cada profissional médico foram aplicados o questionário e as escalas por meio de formulário digital desenvolvido na plataforma Google Forms, não havendo neste identificação, garantido o anonimato e sigilo dos dados coletados.

O recrutamento dos médicos participantes ocorreu por meio dos grupos de WhatsApp e Telegram (aplicativos de mensagens instantâneas) ativos da instituição de saúde. O acesso foi obtido por intermédio do Diretor Geral do Hospital Dr. 
Miguel Couto que recebeu o link do formulário digital e o encaminhou para esses grupos. Antes de visualizar as perguntas, o participante teve acesso ao TCLE eletrônico, onde havia ao final um campo de Consentimento Pós Informação ("Declaro que concordo em participar da pesquisa") e somente foi encaminhado às perguntas após selecionar esse campo. A análise e comparação dos dados foram feitas com a construção de gráficos e tabelas por meio do programa Microsoft Excel.

Pesquisou-se artigos sobre o tema escolhido por meio das bases de dados SCIELO, PUBMED, LILACS e BIREME, publicados em 2011 a 2021 nos idiomas português e inglês, utilizando os seguintes descritores em Ciência da Saúde (DeCS): COVID-19, angústia psicológica, ansiedade, depressão, distanciamento físico, esgotamento psicológico, exercício físico, espiritualidade, pandemias, transtornos mentais e transtorno de estresse pós-traumático. Devido a relevância para o entendimento do trabalho, foram incluídos um artigo do ano de 2004 e outro de 2006, totalizando 20 artigos.

Critérios de inclusão: profissionais médicos que trabalham no Hospital Dr. Miguel Couto e que trabalharam durante a pandemia do Covid-19.

Critérios de exclusão: médicos que foram afastados durante esse período por serem do grupo de risco, os demais profissionais não médicos desse hospital, profissionais de saúde que não trabalham no Hospital Dr. Miguel Couto, participantes que responderam menos de $90 \%$ do questionário e/ou não responderam 100\% das escalas de Triagem de TEPT para Atenção Primária (PC-PTSD), de Transtorno de Ansiedade Generalizada (GAD-2) e Questionário sobre a saúde do paciente (PHO-2) para triagem de TEPT.

Esta pesquisa obedeceu às recomendações da resolução no . 466/12 do Conselho Nacional de Saúde (CNS), que define as diretrizes e normas regulamentadoras de pesquisas envolvendo seres humanos, além de seguir às orientações da Carta Circular $n^{\circ}$ 1/2021 da Comissão Nacional de Ética em Pesquisa (CONEP) para procedimentos em pesquisas com qualquer etapa em ambiente virtual.

Em se tratando de possíveis riscos para a realização desse estudo têm-se constrangimento por parte dos médicos por se tratar de alterações mentais, perda de informações, o que pode gerar exposição dos participantes, e quebra de sigilo. Para minimizar possíveis danos e garantir a confidencialidade e a privacidade dos indivíduos, as pesquisadoras garantiram o anonimato no preenchimento do questionário, assim como a não publicação de resultados individuais. Todos os dados obtidos na pesquisa foram utilizados exclusivamente com finalidades científicas conforme previsto no consentimento do participante. Em caso de eventuais danos, as pesquisadoras se responsabilizarão pelos mesmos.

Parecer do Comitê de Ética Pesquisa: o trabalho foi aprovado pelo CEP UNINOVAFAPI. CAAE: 45519221.7.0000.5210.

\section{Resultados}

Como apresentado na Tabela 1, foram colhidas 29 respostas, sendo 10 do sexo feminino $(34,5 \%)$ e 19 do sexo masculino (65,5\%). Em relação à idade dos profissionais médicos, a maioria tinha entre 20 e 30 anos, totalizando 12 pessoas (41,4\%). Por outro lado, a faixa etária de 51 a 60 totalizou 2 profissionais $(6,9 \%)$, representando a menor quantidade de respostas. Os participantes, em sua maioria, foram pardos $(51,7 \%)$.

Em relação à principal área de atuação, a maioria dos profissionais médicos atuavam como clínico, correspondendo à $51,7 \%$ (15), enquanto 6,9\% (2) eram cirurgiões, $24,1 \%$ (7) intensivistas, $13,8 \%$ (4) urgência e emergência e apenas 3,4\% (1) da área de gestão e administrativo. Ademais, a maioria desses profissionais tinham de 1 a 5 anos de atuação $(55,2 \%)$ e apenas 2 $(6,9 \%)$ tinham mais de 20 anos de serviço. 
Tabela 1: Características sociodemográficas dos participantes $(\mathrm{N}=29)$.

\begin{tabular}{|c|c|c|c|}
\hline \multicolumn{2}{|l|}{ Variável Sociodemográfica } & Frequência & $\%$ \\
\hline \multirow{2}{*}{ Sexo } & Feminino & 10 & $34,5 \%$ \\
\hline & Masculino & 19 & $65,5 \%$ \\
\hline \multirow{3}{*}{ Idade } & 31 a 40 & 11 & $37,9 \%$ \\
\hline & 41 a 50 & 4 & $13,8 \%$ \\
\hline & 51 a 60 & 2 & $6,9 \%$ \\
\hline \multirow[t]{2}{*}{ Raça } & Parda & 15 & $51,7 \%$ \\
\hline & Preta & 1 & $3,4 \%$ \\
\hline \multirow{4}{*}{ Forma de controle de estresse } & Atividade física regularmente & 10 & $34,5 \%$ \\
\hline & Nenhum & 1 & $3,4 \%$ \\
\hline & Outros & 4 & $13,8 \%$ \\
\hline & Psicoterapia & 4 & $13,8 \%$ \\
\hline \multirow{5}{*}{$\begin{array}{l}\text { Principal área de atuação na } \\
\text { Medicina }\end{array}$} & Cirurgião (a) & 2 & $6,9 \%$ \\
\hline & Clínico (a) & 15 & $51,7 \%$ \\
\hline & Gestão ou Administrativo & 1 & $3,4 \%$ \\
\hline & Intensivista & 7 & $24,1 \%$ \\
\hline & Urgência e Emergência & 4 & $13,8 \%$ \\
\hline \multirow{5}{*}{ Tempo que exerce a profissão } & 1 a 5 anos & 16 & $55,2 \%$ \\
\hline & 5 a 10 anos & 6 & $20,7 \%$ \\
\hline & 10 a 15 & 3 & $10,3 \%$ \\
\hline & 15 a 20 & 2 & $6,9 \%$ \\
\hline & Mais de 20 anos & 2 & $6,9 \%$ \\
\hline \multirow{2}{*}{$\begin{array}{l}\text { Diagnosticado com TEPT, } \\
\text { ansiedade ou depressão antes da } \\
\text { pandemia }\end{array}$} & Não & 25 & $86,2 \%$ \\
\hline & Sim & 4 & $13,8 \%$ \\
\hline \multirow{3}{*}{$\begin{array}{l}\text { Uso de medicação para } \\
\text { transtornos mentais }\end{array}$} & Sem resposta & 2 & $6,9 \%$ \\
\hline & Não & 5 & $17,2 \%$ \\
\hline & Não se aplica & 19 & $65,5 \%$ \\
\hline \multirow{4}{*}{$\begin{array}{l}\text { Por quanto tempo usa } \\
\text { medicação }\end{array}$} & Sem resposta & 3 & $10,3 \%$ \\
\hline & Entre 6 meses e 1 an & 2 & $6,9 \%$ \\
\hline & Mais de 1 ano & 1 & $3,4 \%$ \\
\hline & Não se aplica & 23 & $79,3 \%$ \\
\hline \multirow{2}{*}{$\begin{array}{l}\text { Recebeu treinamento para } \\
\text { minimizar riscos de } \\
\text { contaminação }\end{array}$} & Não & 6 & $20,7 \%$ \\
\hline & Sim & 23 & $79,3 \%$ \\
\hline
\end{tabular}

Fonte: Autoras.

Em relação às práticas para controle do estresse (Gráfico 1), apenas 1 participante $(3,4 \%)$ referiu não praticar nenhuma atividade. Atividade física regular e religião e/ou espiritualidade foram as formas mais descritas, correspondendo à 34,5\% (10) das respostas para cada uma. Os participantes também relataram envolvimento com psicoterapia $(13,8 \%)$ e outras formas de controle menos comuns $(13,8 \%)$.

Setenta e nove vírgula três por cento (23) dos profissionais médicos participantes relataram terem recebido treinamento para minimizar riscos de contaminação, enquanto $6(20,7 \%)$ não receberam.

O Gráfico 2 mostra a porcentagem de médicos que classificaram cada fator estressor como severo. Sessenta e cinco por cento dos participantes consideraram ter familiares do grupo de risco (maiores de 60 anos ou com alguma doença crônica) e a escassez de insumos e leitos fatores severamente estressantes. Mais de $40 \%$ dos participantes classificaram as seguintes preocupações como severamente estressantes: carga horária de trabalho, morte de colegas e/ou pacientes, distância social da família e transmitir Covid-19 para familiares. O contato com pacientes com Covid-19 e a falta de diretrizes de tratamento seguras 
também foram fontes importantes de estresse, correspondendo ambas a 37,90\% dos relatos. Em relação aos outros fatores, menos participantes relataram grande preocupação com a falta de EPIs (17,20\%) e renda familiar (13,70\%).

Entre os profissionais médicos do Hospital do Monte Castelo, 4 já tinham diagnóstico de TEPT, ansiedade ou depressão antes da pandemia, correspondendo a 13,8\%. Destes, 3 (10,3\%) fez ou faz uso de medicação durante um período médio de um ano.

Houve uma proporção significativa de participantes com triagem positiva para alterações mentais (Gráfico 3). De acordo com os critérios usados nesta pesquisa por meio das escalas PC-PTSD, GAD-2, PHO-2 para triagem de Transtorno Pós Traumático, ansiedade e depressão, respectivamente, 9 dos participantes foram triados positivos para Transtorno Pós Traumático correspondendo a 45\%, 16 para ansiedade (55\%) e 13 para depressão correspondendo a $45 \%$ do total. Foram desconsiderados aqueles que já tinham diagnóstico anterior para uma das três alterações mentais. Houve uma maior prevalência entre o sexo masculino.

Gráfico 1: Formas de controle de estresse praticadas pelos profissionais médicos do Hospital do Monte Castelo em Teresina/ PI.

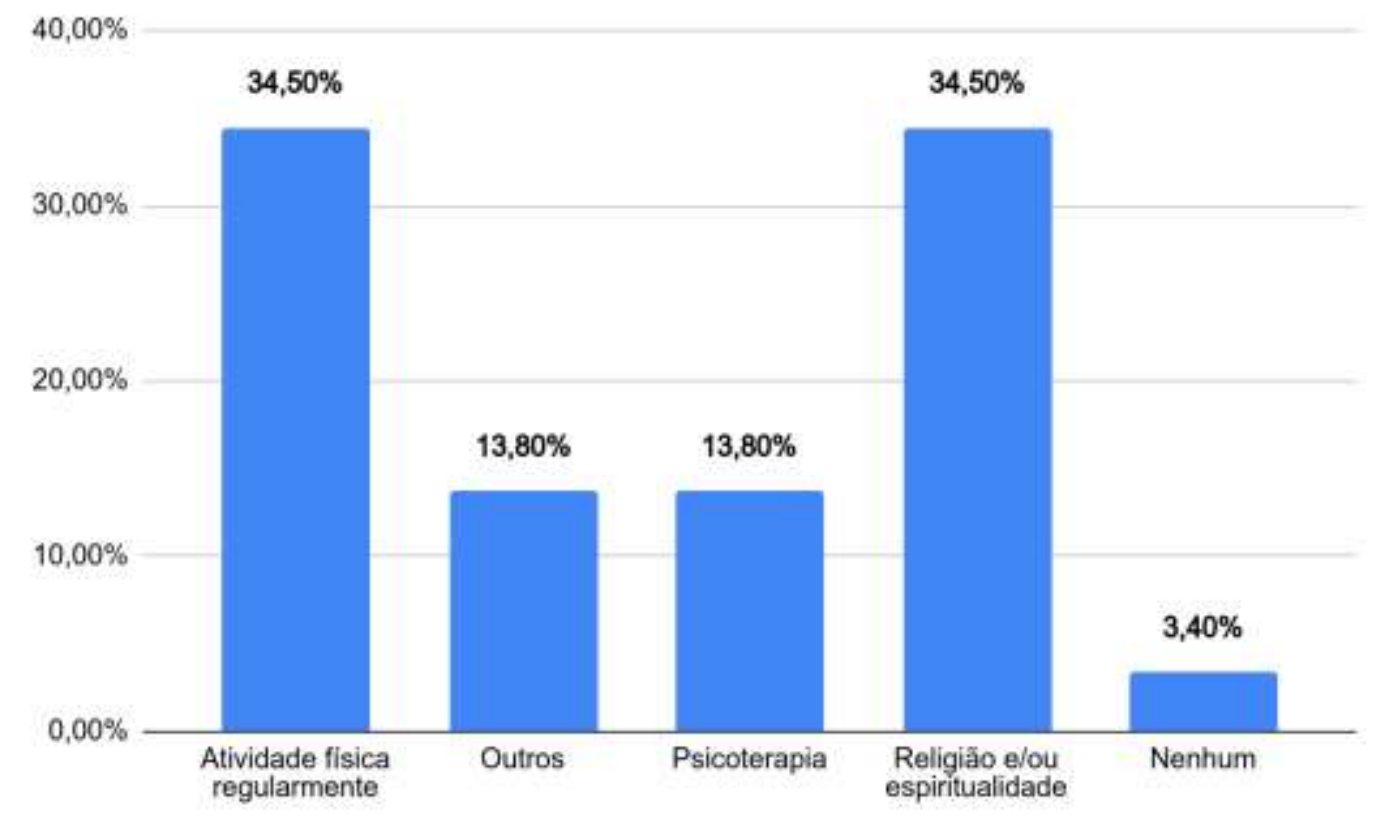

Fonte: Autoras. 
Gráfico 2: Fatores estressores considerados severos pelos profissionais médicos do Hospital do Monte Castelo em Teresina/

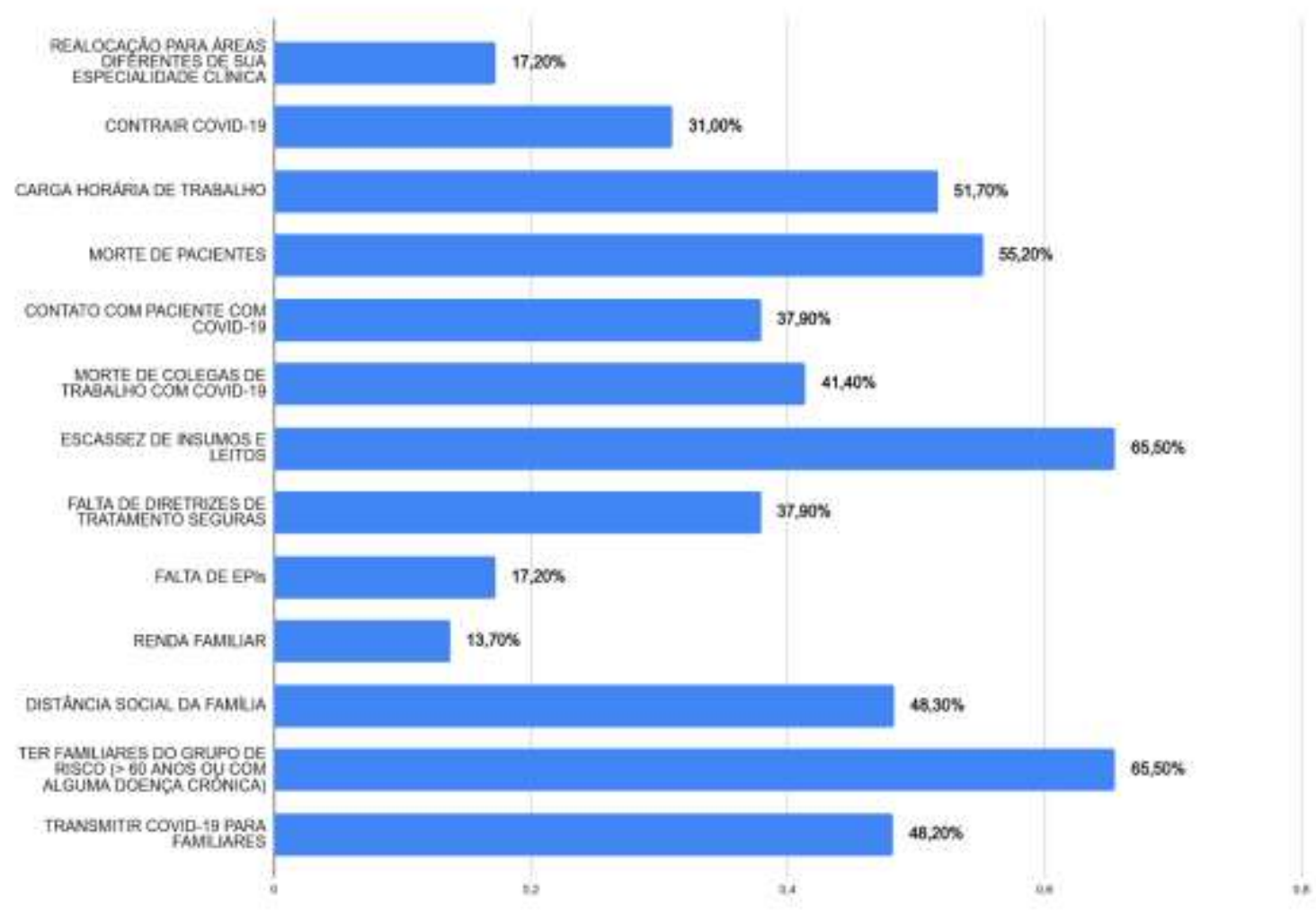

Fonte: Autoras.

Gráfico 3: Porcentagem de profissionais médicos com triagem positiva para ansiedade, depressão e transtorno pós-traumático (TEPT) do Hospital do Monte Castelo em Teresina/ PI.

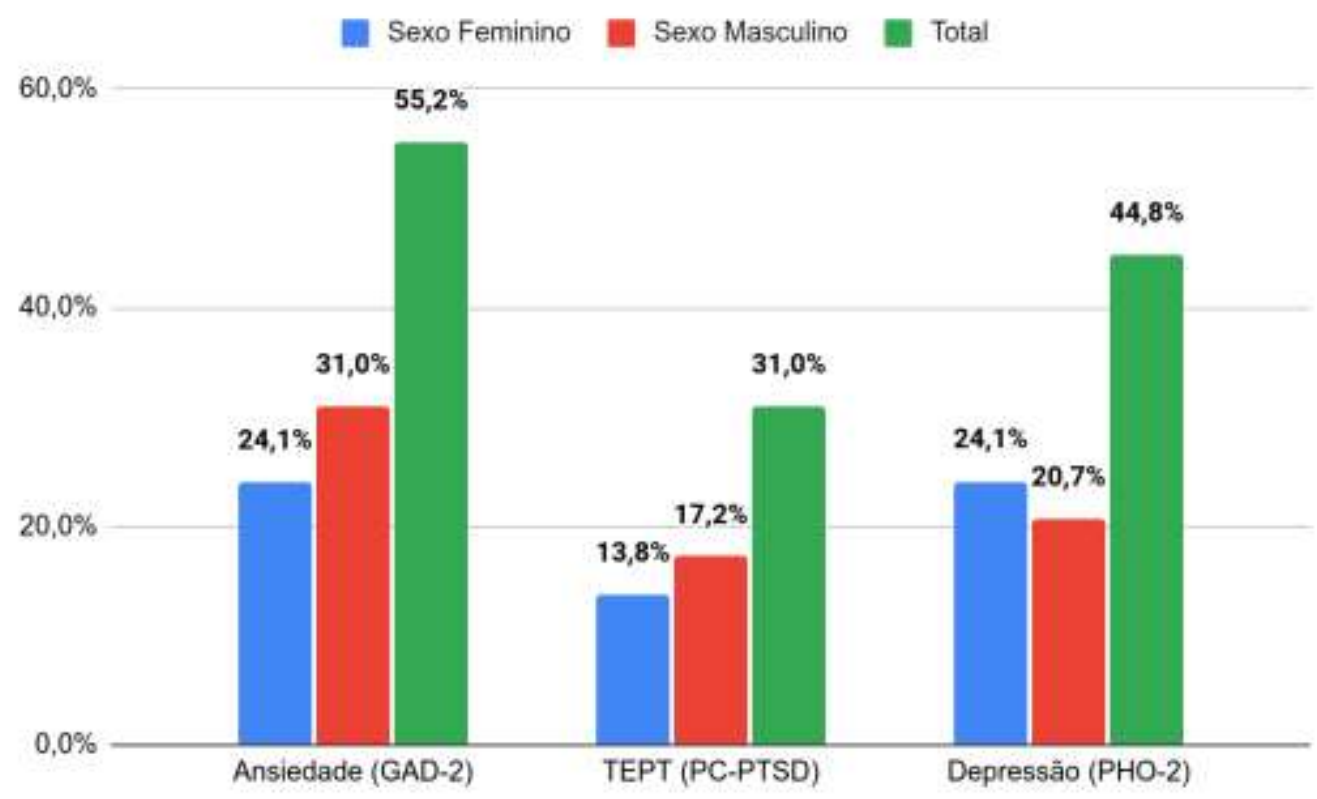

Fonte: Autoras. 
Ao analisar a Figura 1, percebe-se que os participantes pontuaram em duas ou mais alterações mentais, concomitantemente. Foram 8 profissionais com triagem positiva para ansiedade e depressão (27,6\%), 3 para ansiedade e TEPT $(10,3 \%)$ e 5 para ansiedade, depressão e TEPT, o equivalente a 17,2\%. Apenas um participante pontuou para TEPT isoladamente.

Figura 1: Quantidades individuais e de intersecção de profissionais médicos quanto triagem positiva para ansiedade, depressão e transtorno pós-traumático (TEPT) do Hospital do Monte Castelo em Teresina/ PI.

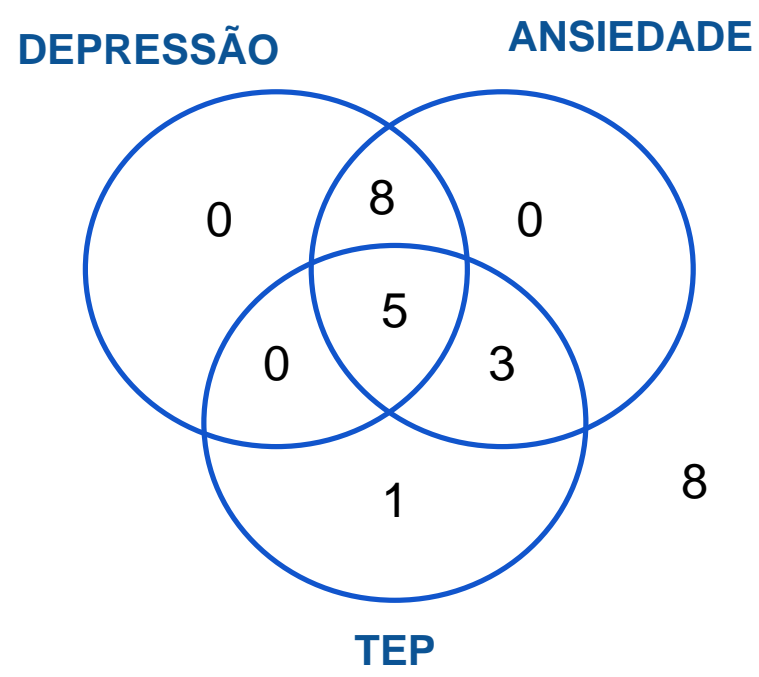

Fonte: Autoras.

\section{Discussão}

Neste estudo transversal realizado em um hospital de referência para COVID-19 em Teresina-PI, mais da metade dos participantes tiveram triagem positiva para ansiedade e quase metade deram resultado positivo para transtorno pós traumático ou para depressão. Destes, a maioria foram do sexo masculino.

Quando avaliado os estressores relacionados à família e preocupações fora do ambiente clínico, ter familiares no grupo de risco (> 65 anos ou com alguma doença crônica) foi a fonte de maior angústia para os médicos entrevistados. Ficando em segundo lugar, numericamente muito próximos, os estressores distanciamento social e medo de transmitir COVID-19 para os familiares.

No ambiente de trabalho o estressor mais prevalente foi a morte de pacientes por COVID-19 e o menos prevalente (apenas $1 / 4$ das respostas) foi a realocação de profissionais médicos para áreas diferentes da sua atuação clínica. Já na avaliação dos estressores relacionados às diretrizes e políticas nacionais, a escassez de insumos e leitos foi o preponderante.

Os resultados obtidos por essa pesquisa são condizentes com outras pesquisas que avaliaram o impacto do COVID-19 na saúde mental dos profissionais que trabalham na linha de frente da pandemia. Um estudo americano feito em um grande centro médico em Nova York durante a pandemia do COVID-19, em que foram entrevistados 657 profissionais da saúde na linha de frente, $57 \%$ dos participantes foram testados positivamente para estresse agudo, $48 \%$ tiveram testes positivos para depressão e 33\% tiveram resultados positivos para ansiedade, o que ratifica o impacto negativo na saúde mental desses profissionais e exalta a importância de medidas que amenizem esses impactos (SHECHTER et al., 2020).

Além disso, uma pesquisa transversal realizada na China, incluindo hospitais da linha de frente do combate ao Covid19 e hospitais que não faziam parte do tratamento específico, avaliou 1257 profissionais de saúde em 34 hospitais em várias regiões da China. Mais de 70\% relataram sofrimento, com 50\% referindo depressão. Os mais severos sintomas foram vistos 
naqueles que trabalhavam em Wuhan (o epicentro da pandemia) e na linha de frente do Covid-19, sendo este considerado um fator de risco isolado para piores resultados de saúde mental, segundo esse estudo (LAI et al., 2020).

Em âmbito nacional, Boni et al (2020) conduziram uma pesquisa na internet entre abril e maio de 2020 entre 3.745 trabalhadores essenciais do Brasil (2842) e da Espanha (903). Houve prevalência significativa de exames positivos para depressão, ansiedade e de ambos, sendo todos superiores no Brasil em comparação com a Espanha. Trabalhar na linha de frente durante a pandemia mostrou-se como um fator que aumentou a probabilidade de resultado positivo para ansiedade e/ou depressão. Além disso, estar em um país com maiores taxas de desigualdade social também demonstrou estar associado a sintomas de ansiedade e depressão, uma vez que o profissional tem que enfrentar, além da doença, os problemas sociais, estruturais e políticos.

Em estudos semelhantes a esse, foram observados um acometimento mais significativo da saúde mental entre as mulheres, com maior número de participantes com sintomas de depressão, ansiedade e estresse agudo. Esse resultado diverge do encontrado no presente estudo, em que observou-se uma maior prevalência de sintomas entre os homens. Todavia, tendo em vista o tamanho da amostra (29 participantes) e a quantidade maior de homens (19), não pode-se inferir que estes tenham sido mais afetados (LAI et al, 2020).

Shanafelt et al. (2020), com base em relatórios de oito sessões de escuta com grupos de profissionais de saúde (envolvendo um total de 69 participantes), informaram as mesmas fontes de estresse avaliadas no presente estudo como mais preponderantes. Acesso a equipamento de proteção individual adequado, exposição ao COVID-19 no trabalho e levar a infecção para casa para sua família, medo concomitante de propagar a infecção no trabalho e falta de acesso a insumos foram algumas das fontes relatadas que, embora possam não afetas a todos, podem enfraquecer a confiança dos profissionais em si mesmos e no sistema de saúde, afetando seu desempenho na assistência à população.

Apesar desse estudo ser transversal, pode-se inferir, com bases em estudos passados, que as alterações mentais podem persistir para além do período de pandemia. De 13 a 26 meses após o surto de síndrome respiratória aguda grave (SARS) ocorrido em 2003, Maunder et al (2006) avaliaram 769 profissionais de saúde em hospitais de Toronto e Hamilton (Canadá) por meio da aplicação de questionários e descobriram níveis significativamente mais altos de burnout, sofrimento psicológico e estresse póstraumático entre esses profissionais. Esse estudo destacou a resiliência dos profissionais de saúde, porém, apesar dessa característica, trabalhar durante o surto de SARS teve um impacto negativo a longo prazo nessas pessoas, o que indica a necessidade de intervenções para reduzir os efeitos adversos de um surto de doenças infecciosas.

Vale ressaltar que esse impacto a longo prazo não se restringe apenas à saúde mental. Segundo Chang et al (2019), episódios de estresses prolongados ativam eixos do sistema neuroendócrino que conduzem ao aumento da frequência cardíaca, do cortisol circulante, norepinefrina e citocinas imunossupressoras que, somados ao sono insatisfatório, estão associados à maior risco cardiovascular, aumento do risco de infecção e recuperação deficiente de feridas.

Também foram avaliados no presente estudo os atuais comportamentos para controle do estresse. A prática regular de atividade física e religião e/ou espiritualidade foram as formas mais descritas, mas os participantes também relataram envolvimento com psicoterapia. Apesar desses resultados serem satisfatórios, levando em consideração a importância da prática de atividades que tragam bem-estar, não foi possível encontrar uma relação direta entre essas práticas e a redução do surgimento de alterações mentais. Apenas um entre os vinte e nove participantes referiu não envolver-se em nenhuma forma de controle de estresse e, mesmo assim, mais de 50\% apresentaram algum comprometimento da sua saúde mental.

Esse resultado diverge do encontrado por Teixeira et al. (2021) que verificou a incidência da espiritualidade entre os profissionais da saúde que atuam na linha de frente do enfrentamento ao Covid-19 em um município do sudoeste da Bahia. Foram entrevistados 147 pessoas e observou-se uma relação positiva entre a espiritualidade e o desenvolvimento de transtornos mentais. Desse modo, o desenvolvimento de transtornos mentais comuns diminuiu à medida que aumentou a espiritualidade, 
demonstrando que a inserção das práticas de espiritualidade/ religiosidade impactam positivamente na saúde mental de cada indivíduo.

Ademais, Tiecker et al. (2020) ao entrevistarem 59 idosos por telefone usando um instrumento multidimensional, que incluía a Escala de Depressão Geriátrica (GDS) e a prática de atividade física, observaram que aqueles participantes que diminuíram a prática de atividade física durante a Covid-19 apresentaram níveis de GDS indicativos de depressão.

Esses achados ratificam que, embora não tenha sido o resultado obtido no presente estudo, a prática regular de atividades para controle de estresse demonstra ser fator protetor para o desenvolvimento de alterações mentais, sendo essencial o estímulo à essa prática, mas sempre respeitando as preferências pessoais, de forma a trazer benefícios físicos e mentais a longo prazo.

\section{Conclusão}

Este estudo teve como objetivo analisar o impacto da pandemia do Covid-19 na saúde mental de profissionais médicos em um hospital público em Teresina-PI, sob a ótica de três principais transtornos: depressão, ansiedade e estresse agudo, por meio da aplicação de escalas para triagem desses transtornos. Foram elencadas várias fontes de estresse relacionados à família e preocupações fora do ambiente clínico, ao ambiente de trabalho e às diretrizes e políticas nacionais.

Percebe-se que os profissionais médicos estão enfrentando problemas substanciais, com grandes proporções de triagem positiva para depressão, estresse agudo e ansiedade. Esses resultados apontam para a permanência de ínfimo oferecimento nos serviços de saúde de suporte psicológico para os profissionais e para a necessidade do desenvolvimento e implementação de políticas públicas efetivas de saúde mental associadas às estratégias de enfrentamento das epidemias e pandemias antes, durante e após o evento, de modo a proteger o bem estar mental e físico dos profissionais médicos visando, também, a permanente assistência de qualidade à população (RAN et al, 2020).

Entende-se a complexidade do tema proposto neste artigo, para tanto, assume-se a necessidade e a importância do desenvolvimento de novos trabalhos, levando-se em conta a possibilidade de uso de tamanho de amostra maiores, a fim de melhorar a replicabilidade do estudo. Também poderia ser interessante um período de acompanhamento mais longo dos indivíduos da amostra, utilizando uma metodologia longitudinal, com o intuito de avaliar tanto os transtornos mentais abordados no presente estudo quanto outros transtornos não abordados, por exemplo a psicose, e seus desdobramentos a mais longo prazo, tendo em vista que a literatura traz o estresse como um gatilho considerável nos processos de adoecimento mental, de forma geral.

Ademais, seria válido desmembrar o estudo utilizando parâmetros para comparar grupos, de forma a analisar como cada variável investigada afeta individualmente a saúde mental dos profissionais, como num estudo retrospectivo. Um exemplo seria comparar um grupo de profissionais que trabalharam em urgência/emergências e Unidades de Terapia Intensiva com profissionais que exerceram a medicina exclusivamente na modalidade ambulatorial durante a pandemia do SARS-COV-2, ou comparar dois grupos de profissionais que trabalharam concomitantemente no período da pandemia, um que tenha desenvolvido antes ou durante a pandemia, formas de controle de estresse (seja atividade física, psicoterapia, religião e/ou espiritualidade ou outras) e um segundo grupo que não tenha recorrido a nenhuma forma de controle de estresse.

Com esses esforços, pretende-se, cada vez mais, construir subsídios científicos sólidos que contribuam e apoiem a redução da forte associação que a saúde mental tem com o cenário patológico estrutural das noções de qualidade de vida e consolidá-la no viés de promoção da saúde. Espera-se que essas descobertas influenciem, não somente o Hospital do Monte Castelo, mas também as políticas de promoção e proteção dos profissionais médicos de outras instituições enquanto passam por essa ou por futuras crises de saúde pública. 


\section{Referências}

Castro, L. d. S. (2011). Adaptação e validação do índice de gravidade de insônia (IGI): caracterização populacional, valores normativos e aspectos associados.

Chang B. P. (2019). Can hospitalization be hazardous to your health? A nosocomial based stress model for hospitalization. General hospital psychiatry, 60, 8389. https://doi.org/10.1016/j.genhosppsych.2019.07.014.

De Boni, R. B., Balanzá-Martínez, V., Mota , J. C., Cardoso, T. D. A., Ballester, P., Atienza-Carbonell, B., Bastos, F. I., \& Kapczinski, F. (2020). Depression, Anxiety, and Lifestyle Among Essential Workers: A Web Survey From Brazil and Spain During the COVID-19 Pandemic. JMIR Publications, 22(10), 1-16. https://doi.org/10.2196/22835.

Gaya, C. M. (2011). Estudo de validação de instrumentos de rastreamento para transtornos depressivos, abuso e dependência de álcool e tabaco. Tese de Doutorado, Faculdade de Medicina de Ribeirão Preto, Universidade de São Paulo, Ribeirão Preto. doi:10.11606/T.17.2011.tde-26092011-144558. www.teses.usp.br.

Kang, L., Li, Y., Hu, S., Chen, M., Yang, C., Yang, B. X., Wang, Y., Hu, J., Lai, J., Ma, X., Chen, J., Guan, L., Wang, G., Ma, H., \& Liu, Z. (2020). The mental health of medical workers in Wuhan, China dealing with the 2019 novel coronavirus. The lancet. Psychiatry, 7(3), e14. https://doi.org/10.1016/S22150366(20)30047-X.

Kroenke, K., Spitzer, R. L., Williams, J. B., Monahan, P. O., \& Löwe, B. (2007). Anxiety disorders in primary care: prevalence, impairment, comorbidity, and detection. Annals of internal medicine, 146(5), 317-325. https://doi.org/10.7326/0003-4819-146-5-200703060-00004.

Lai, J., Ma, S., Wang, Y., Cai, Z., Hu, J., Wei, N., Wu, J., Du, H., Chen, T., Li, R., Tan, H., Kang, L., Yao, L., Huang, M., Wang, H., Wang, G., Liu, Z., \& Hu, S. (2020). Factors Associated With Mental Health Outcomes Among Health Care Workers Exposed to Coronavirus Disease 2019. JAMA network open, 3(3), e203976. https://doi.org/10.1001/jamanetworkopen.2020.3976.

Maunder, R. G., Lancee, W. J., Balderson, K. E., Bennett, J. P., Borgundvaag, B., Evans, S., Fernandes, C. M., Goldbloom, D. S., Gupta, M., Hunter, J. J., McGillis Hall, L., Nagle, L. M., Pain, C., Peczeniuk, S. S., Raymond, G., Read, N., Rourke, S. B., Steinberg, R. J., Stewart, T. E., VanDe Velde-Coke, S., ... Wasylenki, D. A. (2006). Long-term psychological and occupational effects of providing hospital healthcare during SARS outbreak. Emerging infectious diseases, 12(12), 1924-1932. https://doi.org/10.3201/eid1212.060584.

Meyerowitz, E. A., Richterman, A., Gandhi, R. T., \& Sax, P. E. (2021). Transmission of SARS-CoV-2: A Review of Viral, Host, and Environmental Factors. Annals of internal medicine, 174(1), 69-79. https://doi.org/10.7326/M20-5008.

Ministério Da Saúde. (2021). Painel Coronavírus. Coronavírus Brasil. https://covid.saude.gov.br/.

Organização Mundial De Saúde. (2021). WHO Coronavirus Disease (COVID-19) Dashboard. https://covid19.who.int/.

Ornell, F., Schuch, J. B., Sordi, A. O., \& Kessler, F. (2020). "Pandemic fear" and COVID-19: mental health burden and strategies. Revista brasileira de psiquiatria (Sao Paulo, Brazil : 1999), 42(3), 232-235. https://doi.org/10.1590/1516-4446-2020-0008.

Pereira A. S., Shitsuka, D. M., Parreira, F. J., Shituka, R. (2018). Metodologia da pesquisa científica. UFSM.

Prins, A., Ouimette, P., Kimerling, R., Cameron, R. P., Hugelshofer, D. S., Shaw-Hegwer, J., Thrailkill, A., Gusman, F. D., \& Sheikh, J. I. (2003). The primary care PTSD screen (PC-PTSD): Development and operating characteristics. Primary Care Psychiatry, 9(1), 9-14. https://doi.org/10.1185/135525703125002360.

Ran, L., Chen, X., Wang, Y., Wu, W., Zhang, L., \& Tan, X. (2020). Risk Factors of Healthcare Workers With Coronavirus Disease 2019: A Retrospective Cohort Study in a Designated Hospital of Wuhan in China. Clinical infectious diseases : an official publication of the Infectious Diseases Society of America.

Secretaria De Estado Da Saúde. (2021, November 9). Painel Epidemiológico Covid-19. https://datastudio.google.com/reporting/a6dc07e9-4161-4b5a-9f2a6f9be486e8f9/page/2itOB.

Shanafelt, T., Ripp, J., \& Trockel, M. (2020). Understanding and Addressing Sources of Anxiety Among Health Care Professionals During the COVID-19 Pandemic. JAMA, 323(21), 2133-2134. https://doi.org/10.1001/jama.2020.5893.

Shechter, A., Diaz, F., Moise, N., Anstey, D. E., Ye, S., Agarwal, S., Birk, J. L., Brodie, D., Cannone, D. E., Chang, B., Claassen, J., Cornelius, T., Derby, L., Dong, M., Givens, R. C., Hochman, B., Homma, S., Kronish, I. M., Lee, S., Manzano, W., ... Abdalla, M. (2020). Psychological distress, coping behaviors, and preferences for support among New York healthcare workers during the COVID-19 pandemic. General hospital psychiatry, 66, 1-8. https://doi.org/10.1016/j.genhosppsych.2020.06.007.

Teixeira, V. M. d. S., Alves, H. R., Silva, L. F. d., Souza, A. A., Filho, W. d. F., \& Martins, A. M. E. d. B. L. (2021). Espiritualidade entre os profissionais da saúde que atuam na linha de frente do enfrentamento à COVID-19, em um município do Sudoeste da Bahia e sua correlação com a qualidade de vida, transtornos mentais e medo da COVID. Research, Society and Development, 10(10).

Tiecker, A. P., Aguirre, F., Machado, J., \& Bós, Ângelo J. (2020). Atividade física e sintomas depressivos em nonagenários e centenários antes e durante o isolamento social. Revista Brasileira De Ciências Do Envelhecimento Humano, 17(2). https://doi.org/10.5335/rbceh.v17i2.11948.

Xiang, Y. T., Jin, Y., \& Cheung, T. (2020). Joint International Collaboration to Combat Mental Health Challenges During the Coronavirus Disease 2019 Pandemic. JAMA psychiatry, 77(10), 989-990. https://doi.org/10.1001/jamapsychiatry.2020.1057. 